\title{
Hommage
}

\section{À notre collègue et ami, Pierre Gangloff}

La mort tombe dans la vie comme une pierre dans un étang : d'abord, éclaboussures, affolements dans les buissons, battements d'aile et fuites en tout sens. Ensuite, grands cercles sur l'eau, de plus en plus larges. Enfin le calme à nouveau, mais pas du tout le même silence qu'auparavant, un silence, comment dire : assourdissant.

Christian Bobin

Pierre,

Après mûre réflexion, j'ai finalement décidé que cet hommage devait s'adresser à toi. Peut-être aussi parce que cela me donne la possibilité de faire comme si je te parlais, une dernière fois.

Il est indispensable, bien que beaucoup d'entre nous le connaisse, de rappeler ton parcours. Tu es né en Alsace dans une famille aimante, dont tu étais le petit dernier et le seul fils. Tu as grandi entouré de tes parents et de tes soeurs, et tu as choisi d'être chirurgien-dentiste. Tu as effectué ton cursus à la Faculté de Chirurgie Dentaire de Nancy, et tu as décidé de passer l'internat. Bien sûr, le succès fut au rendez-vous et tu as fait partie de la troisième promotion d'internes en Odontologie. Tu es resté en Lorraine pour effectuer ton troisième cycle, et tu as rencontré Anne. Tu as été assistant hospitalouniversitaire et pendant cette période, tu as soutenu ta thèse de Doctorat en Neurosciences. Après plusieurs années passées au CHU de Nancy auprès du regretté Christian Wang, ton mentor comme tu l'appelais, tu as quitté Nancy pour Metz. Mais seulement professionnellement d'abord, puisque tu as continué à vivre avec Anne dans cet appartement d'étudiant trop petit, tout près de la fac. Enfin, il y a bientôt 6 ans, tu as quitté la cité ducale pour t'installer en Moselle, à Peltre. Les petits sont arrivés, Nicolas en 2011 et Alexandre l'an dernier. Tu étais un père comblé et ce rôle te convenait à merveille.

Depuis plusieurs années tu exerçais ton art dans le service d'Odontologie du CHR Metz-Thionville, à l'Hôpital Bonsecours puis à Mercy. Tu y étais épanoui, très apprécié, et tu es devenu indispensable. Le Docteur Eric Gérard, responsable du service, avait même prévu de t'en confier les rênes... mais tu n'auras pas eu le temps de prendre cette responsabilité.

Tout comme tu avais aimé être interne, j'ai aimé l'être. Et j'ai aimé l'être parce que j'ai eu la chance d'être formée par des gens comme toi. La pratique de la chirurgie orale était l'une de tes passions, mais tu n'as jamais délaissé le versant universitaire. Tu dispensais des cours à la Faculté d'0dontologie de Nancy, tu étais un membre actif de la SFCO, tu publiais (17 publications internationales, sans compter les communications, les recommandations...), tu lisais. Chance pour moi, pour nous les internes, car tu avais toujours réponse à tout! Quel bonheur de travailler avec toi au quotidien, d'écouter tes conseils, de t'observer travailler, et d'entendre chaque jour ton humour et tes rires. Avec toi l'ambiance était toujours bonne, même quand c'était difficile, même quand il y avait beaucoup de patients, car jamais tu ne les délaissais. Tu connaissais les gens, tu les aimais. Tu savais leur parler, les mettre à l'aise, les rassurer. Je pense en particulier aux patients de l'Institut de Cancérologie de Lorraine où tu étais praticien attaché, qui te regrettent tant. Comme eux je regrette ton absence, tes expressions, tes mimiques, ta gentillesse et ton charisme.

Qu'Anne et Alexandre trouvent la force de vivre et d'avancer sans toi et Nicolas, veille bien sur eux ; mais aussi sur nous, les enfants de la chirurgie orale, qui te sommes si reconnaissants de ce que tu nous as transmis, et à qui tu manques profondément.

Julie Guillet

\footnotetext{
* Correspondance : j.guillet@chu-nancy.fr

This is an Open Access article distributed under the terms of the Creative Commons Attribution License (http://creativecommons.org/licenses/by/4.0), which permits unrestricted use, distribution, and reproduction in any medium, provided the original work is properly cited
} 